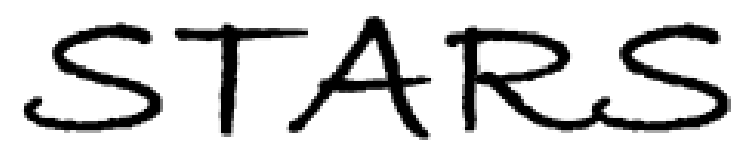

University of Central Florida

STARS

$1-1-1991$

\title{
Enhancement Of Excited-State Population Of Magnesium-Ions Upon Decay Of Autoionizing Resonances
}

\author{
S. B. Whitfield \\ University of Central Florida \\ C. D. Caldwell \\ University of Central Florida
}

M. O. Krause

Find similar works at: https://stars.library.ucf.edu/facultybib1990

University of Central Florida Libraries http://library.ucf.edu

This Article is brought to you for free and open access by the Faculty Bibliography at STARS. It has been accepted for inclusion in Faculty Bibliography 1990s by an authorized administrator of STARS. For more information, please contact STARS@ucf.edu.

\section{Recommended Citation}

Whitfield, S. B.; Caldwell, C. D.; and Krause, M. O., "Enhancement Of Excited-State Population Of Magnesium-Ions Upon Decay Of Autoionizing Resonances" (1991). Faculty Bibliography 1990s. 370. https://stars.library.ucf.edu/facultybib1990/370

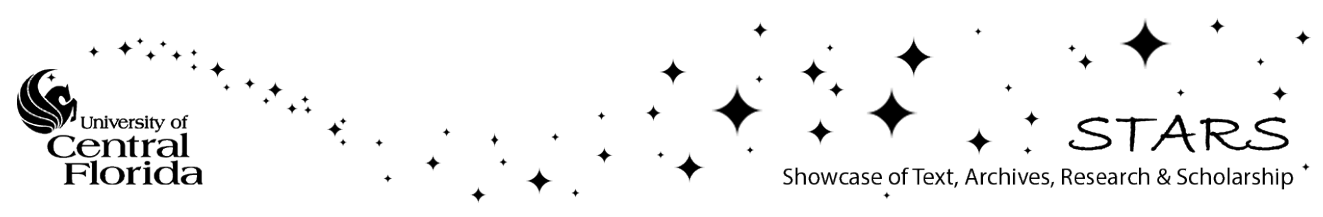




\title{
Enhancement of excited-state population of magnesium ions upon decay of autoionizing resonances
}

\author{
S. B. Whitfield and C. D. Caldwell \\ Department of Physics, University of Central Florida, Orlando, Florida 32816 \\ M. O. Krause \\ Chemistry Division, Oak Ridge National Laboratory, Oak Ridge, Tennessee 37831-6201
}

(Received 24 September 1990)

\begin{abstract}
Decay of autoionizing resonances in atoms often leads to preferential production of excited states of the resulting ion. The population of these states can exceed the population of the ground state by more than an order of magnitude. We report measurements of the branching ratios for $\mathrm{Mg}^{+}$ions resulting from the decay of selected autoionizing levels. These derive either from single excitation of a $2 p$ electron or from two-electron excitations. For excited states of the type $2 p^{5}\left({ }^{2} P_{1 / 2,3 / 2}\right) 3 s^{2} n s$, $n=4$ or 5 , production of $\mathrm{Mg}^{+}$ions having the final configuration $2 p^{6} n s$ is the predominant decay feature. For $n>5$, the predominant decay feature is the $2 p^{6}(n+1) s$ configuration, indicating that shakeup is very strong in the decay of the excited state. For excited states of the type $2 p^{5}\left({ }^{2} P_{1 / 2}\right) 5 d$ and $2 p^{5}\left({ }^{2} P_{3 / 2}\right) 6 d$, the decay behaves in a manner similar to that of the $n s$ states. However, for the $2 p^{5}\left({ }^{2} P_{1 / 2}\right) 6 d$ state, the most prominent decay channel appears to be the $2 p^{6} 11 d$ state, which leads to the emission of an electron with the same kinetic energy as the $L_{2}-M_{1} M_{1}$ Auger line.
\end{abstract}

\section{INTRODUCTION}

Over the past ten years there has been significant experimental interest in the dynamics of photoionization of the alkaline-earth elements (group II) in the atomic state. Much of this work has concentrated on the photoionization cross sections of the valence electrons and their correlation satellites through the autoionizing $n l^{-1}(n+1) s^{2} n^{\prime} l \pm 1$ states (where $n$ represents the principal quantum number of the second outermost shell, and $\left.n^{\prime} \geq n+1\right)$. However, a substantial amount of this work has also focused on the enhancement of the main photoline and its correlation satellites following the subsequent decay of the photoexcited $n l^{-1}(n+1) s^{2} n^{\prime} l \pm 1$ states via autoionization. The first experimental study of photoexcitation and decay on a group-II element using a synchrotron was carried out on $\mathrm{Ba}^{1}$ In that work the authors investigated the decay channels of the photoexcited configurations $5 p^{5} 6 s 5 d^{2}\left({ }^{1} P_{1}\right)$ and $5 p^{5} 6 s 5 d 6 d\left({ }^{1} P_{1}\right)$. They found that in the second instance, the excited $6 d$ electron did not participate strongly in the autoionizing decay, which led to an enhancement in the production of an excited state of the $\mathrm{Ba}^{+}$ion relative to the ground state of the ion. Numerous other experimental and theoretical investigations involving the $5 p$ resonances in $\mathrm{Ba}$ have been reviewed by Kobrin et al. ${ }^{2}$

Similar investigations have also been carried out on $\mathrm{Sr},{ }^{3,4} \mathrm{Ca},{ }^{5,6}$ and most recently $\mathrm{Be}^{7}$ In all cases it was observed that at certain resonance energies the decay of the excited neutral led to the population of excited-state levels in the ion which were equal to or of greater magnitude than that of the ground-state population. This enhancement of the population of an excited state of the ion over a ground state was most strikingly apparent in the case of Be. In this case it was found that the decay of the $\mathrm{Be}$
$1 s 2 s^{2} 2 p\left({ }^{1} P_{1}\right)$ state to the $\mathrm{Be}^{+} 1 s^{2} 2 p$ state was 56 times stronger than the decay to the $\mathrm{Be}^{+} 1 s^{2} 2 s$ state.

Surprisingly, there have been virtually no experimental investigations of photoionization of $\mathrm{Mg}$ focusing on the excitation and ionization of the $2 p$ electrons. Hausmann et $a l .{ }^{8}$ have measured photoelectron and Auger electron angular distributions; however, this was done at $80 \mathrm{eV}$, well above the ionization thresholds of the $2 p_{1 / 2,3 / 2}$ levels and hence, far above the region for $2 p \rightarrow n s(d)$ excitations. An electron impact experiment was carried out by Breuckmann, Schmidt, and Schmitz. ${ }^{9}$ Electron impact spectra are inherently more complicated since both excitation and ionization lines will be simultaneously present. In addition, since numerous excitation resonances can be reached by one sufficiently energetic electron beam, the task of delineating the various decay channels and generalizing the behavior of the decay dynamics can be rather complicated. Finally, several detailed photoabsorption experiments have been carried out in the region of $2 p$ excitation and ionization, the most recent by Ederer, Lucatorto, and Mehlman. ${ }^{10}$

Theoretically, the photoionization cross section of the $3 s$ electron of atomic $\mathrm{Mg}$ through the $2 s \rightarrow n s(d)$ resonances has been calculated. ${ }^{11,12}$ However, due to rapid fluctuations in the parameters of their multichannel quantum defect theory (MQDT), Deshmuhk and Manson ${ }^{11}$ were unable to calculate the $3 s$ cross section in the region of the $2 p \rightarrow n s(d)$ excitations except very near the $2 p_{3 / 2}$ threshold and between the $2 p_{3 / 2}$ and $2 p_{1 / 2}$ thresholds. There are apparently no theoretical calculations for the cross sections of any of the correlation satellites or for the branching ratios associated with the decay of a given excited state of the neutral.

Magnesium, because of its rather simple dynamics, is ideally suited to a detailed experimental investigation of 
the nature carried out on the other group-II elements. Unlike $\mathrm{Ba}, \mathrm{Sr}$, and even $\mathrm{Ca}$, there are no other series limits which exist below the $2 p_{1 / 2,3 / 2}$ thresholds. ${ }^{10}$ In $\mathrm{Sr}$ there are five two-electron limits below the $4 p_{1 / 2,3 / 2}$ thresholds, ${ }^{13}$ while in Ca there are four two-electron limits below the $3 p_{1 / 2,3 / 2}$ thresholds. ${ }^{5}$ This leads to numerous additional structure in the cross sections of the main photoline and its correlation satellites, significantly complicating the assignments of the excited states of the neutral. In addition, this can also lead to autoionization of the excited neutral into the continua of one of the aforementioned sub- $n p_{1 / 2,3 / 2}$ thresholds. The resulting ion can then Auger decay, giving rise to additional lines in the electron spectra of the ion which are not due to autoionization. ${ }^{3,5}$

In this paper, we report for the first time, as far as we are aware, a detailed experimental analysis of the branching ratios for the autoionizing decay of 11 different excited states of neutral $\mathrm{Mg}$, most arising from the excitation of a $2 p_{1 / 2,3 / 2}$ electron to an $n s(d)$ state. We also present the first partial photoionization cross sections for the $3 \mathrm{~s}$ line and several of its correlation satellites in the energy region of the $2 p \rightarrow n s(d)$ excitations. Finally, we will comment on the rather surprising tendency for the $2 p_{1 / 2}^{-1} 3 s^{2} 6 d$ excited state to preferentially decay to the $2 p^{6} 11 d$ state, thereby emitting an electron of the same energy as the $L_{2}-M_{1} M_{1}$ Auger line.

\section{EXPERIMENT}

This experiment was carried out at the 3-m toroidal grating monochromator (TGM) at the University of Wisconsin Synchrotron Radiation Center. The electron spectrometer which we used for all measurements has been described in detail elsewhere. ${ }^{14,15}$ Radiation from the 3-m TGM was directed through a glass capillary into a vapor oven where a source of $\mathrm{Mg}$ atoms was created by evaporation of the solid. The design of the vapor oven and electron source cell used in this experiment differs from the earlier arrangement. ${ }^{14,15}$ In this "internal" oven the electron source region is attached to the vapor oven in such a way that the interaction between the light and the vapor takes place inside the oven, rather than with an effusive beam emanating from the oven into the source cell. The oven was typically operated at about $580^{\circ} \mathrm{C}$, providing a vapor pressure on the order of $(2-3) \times 10^{-3}$ Torr. The electrons were energy analyzed in a spherical sector plate electrostatic analyzer having a resolution of $1 \%$ of the pass energy. The analyzer was set to the "magic angle" so as to avoid the influence of any angular distributions on the measured intensities. In our case, this angle was found to be $55.5^{\circ}$ with respect to the major axis of the electric field vector of the incident radiation. We determined this value by measuring the polarization $p$ of the radiation exiting from the 3-m TGM and using the relation $\theta_{m}=\left(\frac{1}{2}\right) \cos ^{-1}[1 /(3 p)]$. The polarization was determined from angular distribution measurements of He photoelectrons, for which the asymmetry parameter $\beta$ can be assumed to be 2.0. We found $p=0.77(3)$.

We employed three different methods to record our electron spectra: the constant-ionic-state (CIS) mode, in which spectra are recorded by simultaneously scanning the incident photon energy and the accelerating (retarding) voltage of the source cell (in this case the oven) so as to always observe electrons which correspond to the same final ionic state; the constant-final-state (CFS) mode where spectra are recorded in the same manner as the CIS mode except that the source cell is not scanned along with the photon energy but remains at a constant voltage so as to always observe electrons with the same kinetic energy; and the photoelectron spectrometry (PES) mode, in which spectra are recorded at a fixed photon energy while scanning the accelerating (retarding) voltage of the source cell or the analyzer plates. PES spectra were stored in a multichannel scalar which could be multiplexed, while CIS and CFS spectra were stored directly into a Microvax computer.

The resolution of CIS and CFS scans is determined solely by the bandpass of the monochromator. In our case entrance and exit slit settings of $0.381 \mathrm{~mm}$ were found to be an excellent compromise between monochromator resolution and flux. By fitting the $\mathrm{He} 2 s 2 p$ autoionization resonance at $60.13 \mathrm{eV}$ (Ref. 16) [the natural width $\Gamma=0.038 \mathrm{eV}$ (Ref. 16)] to a Shore profile, ${ }^{17}$ including convolution with the monochromator bandpass, we determined the bandpass from a best fit to be $0.30(2) \AA$. In order to minimize the effects of second-order radiation diffracted from the 3-m TGM, an Al filter was inserted into the beam. Changes in photon flux during the course of the CIS or CFS scan resulting from the decay of the beam current and changes in the monochromator throughput at different photon energies were corrected by dividing out a $\mathrm{Ni}$ mesh current which was simultaneously recorded along with each scan. The Ni mesh was located beyond the last optics of the 3-m TGM just prior to the entrance of the experimental setup. To account for any decrease in light intensity during the course of the scan which might arise from deposition of magnesium on the light collimating capillary, PES spectra were recorded at a suitable resonance energy before and after the scan. After normalizing these spectra to beam current and counting time, any difference in intensity between the two PES spectra could then be used to correct the CIS or CFS spectrum. In most cases, this correction was found to be negligible. In addition, PES scans were recorded at the initial and final photon energies of the scan to determine the background of the CIS or CFS scan. This background was then subtracted out from the raw data. In several cases, however, off-resonance cross sections of the $3 s$ correlation satellites were so small that PES spectra before and after the CIS scan could not be obtained in a reasonable amount of time. In such cases, no background correction to the CIS scan was made. Fortunately, direct photoionization of these satellites is so small compared to their resonant enhancement via autoionization (see Fig. 2) that such a background correction is virtually unnecessary.

Since all PES spectra were recorded while scanning the voltage on the source cell, the pass energy for each scan at every channel was constant. Thus, excluding any lens effects, which in the case of the present experimental setup only arise for acceleration (retardation) voltages which 
are very large compared to the initial kinetic energy of the detected electrons, the transmission function of the analyzer was constant for every channel of the scan. We verified a negligible dependence of the transmission by measuring the intensity of a given photoline as a function of acceleration voltage. Hence, after subtracting the background, the integrated counts under any given peak in the PES spectrum could be taken to be the relative cross section of that feature for the photon energy at which the spectrum was recorded.

Normalization to a common relative scale between the various PES spectra at different photon energies, and between the CIS and PES spectra, was accomplished in a self-consistent manner as follows. The peak height of the $3 s$ photoline in Fig. 4(e) was arbitrarily chosen to be 100 . This photon energy corresponded to the greatest enhancement of the $3 \mathrm{~s}$ line. Similarly, the peak height of the $3 s$ cross section [Fig. 2(a)] at the photon energy at which the spectrum in Fig. 4(e) was recorded was also set to 100 . Hence, the peak height of the $3 s$ line for each resonance energy [Fig. 2(a)] could then be used to scale all the other PES spectra, which in turn could then be used to scale the remaining CIS spectra. As a cross check, the peak height of a given feature at a given photon energy was compared with its value determined in the normalized PES spectrum and in the normalized CIS spectrum. In nearly all cases, excluding the normalization points, which are of course equal, both spectra gave the same result within their error limits. In most cases this amounted to less than $10 \%$.

Integrated intensities and positions of the various PES features were determined by fitting the data with multiple Pearson-7 functions ${ }^{18}$ and a sloping or exponential background. The four-parameter Pearson-7 function can be continuously varied from a pure Lorentzian line shape to a pure Gaussian line shape, making it ideally suited to fitting Voigt profiles.

\section{RESULTS AND DISCUSSION}

\section{A. Overview}

The premise of this experiment was to excite a $2 p_{1 / 2,3 / 2}$ core electron to some excited state [typically an $n s(d), n \geq 3$ level], and then observe how the decay of that state (autoionization) populated various exit channels. This is shown schematically in Fig. 1 for a $2 p_{3 / 2} \rightarrow 4 s$ excitation. A systematic measurement of the branching ratios for the decay of the various excited states into their respective exit channels provides an excellent test of the spectator model. This model has been strongly corroborated in the rare gases $\mathrm{Ar},{ }^{19} \mathrm{Kr},{ }^{20,21}$ and $\mathrm{Xe},{ }^{22}$ and appears to be valid in most cases involving the alkaline earth elements, except for radium, which has not been experimentally investigated.

There are two possible branches of decay for the excited states investigated in this paper. Consider the present case with $\mathrm{Mg}$ in the excited state $1 s^{2} 2 s^{2} 2 p^{5} 3 s^{2} n s(d)$. The two possible transitions are

$$
1 s^{2} 2 s^{2} 2 p^{5} 3 s^{2} n s(d) \rightarrow 1 s^{2} 2 s^{2} 2 p^{6} n s(d) \varepsilon l
$$

and

$$
1 s^{2} 2 s^{2} 2 p^{5} 3 s^{2} n s(d) \rightarrow 1 s^{2} 2 s^{2} 2 p^{6} 3 s \varepsilon^{\prime} l^{\prime \prime},
$$

a spectator and a participator transition, respectively. According to the spectator model, the probability for the excited electron to participate in the decay of the core hole is significantly smaller than if it remains a spectator during the decay process. Qualitatively this is very clear. One would expect that the probability of finding a $3 s$ electron in the vicinity of the empty $2 p$ orbital to be more likely than finding an $n s(d)$ electron in the vicinity of the empty $2 p$ orbital, since on average the $n s(d)$ electron will be found farther from the nucleus than the $3 s$ electron. Hence, the $n s(d)$ electron is not as likely to participate in the decay of the excited neutral as would the $3 \mathrm{~s}$ electron.

PES spectra were recorded at twelve different photon energies: eleven at autoionization resonances and one off resonance. In order to determine the appropriate photon energies at which to record the various PES spectra, several CIS spectra were taken. These spectra, which are

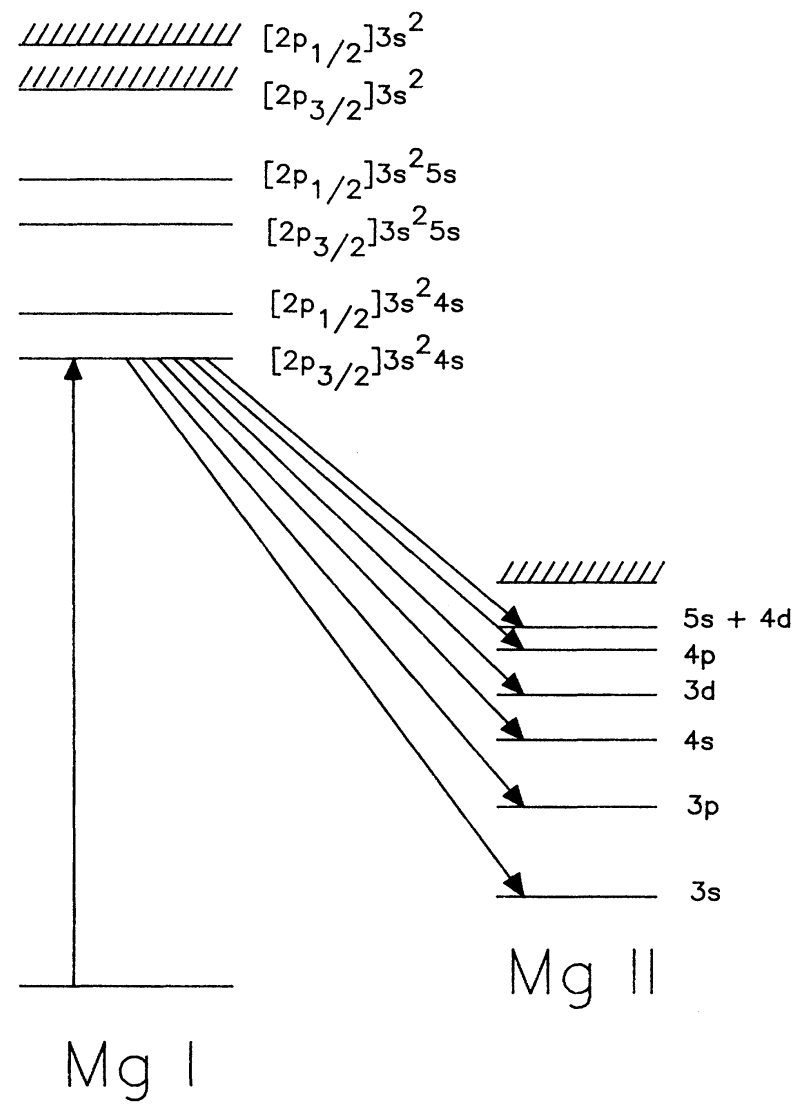

FIG. 1. Simplified energy-level diagram of selected levels of $\mathrm{Mg}$ I and $\mathrm{Mg}$ II. Photoexcitation from the ground state of $\mathrm{Mg}$ I to the $(\mathrm{Ne})\left[2 p_{3 / 2}\right] 3 s^{2} 4 s$ excited state is indicated by the vertical arrow. Decay from the excited state into the various levels of the ion is indicated by the diagonal arrows. Square brackets indicate holes. All configurations are based on a Ne core. This figure is not drawn to scale. 

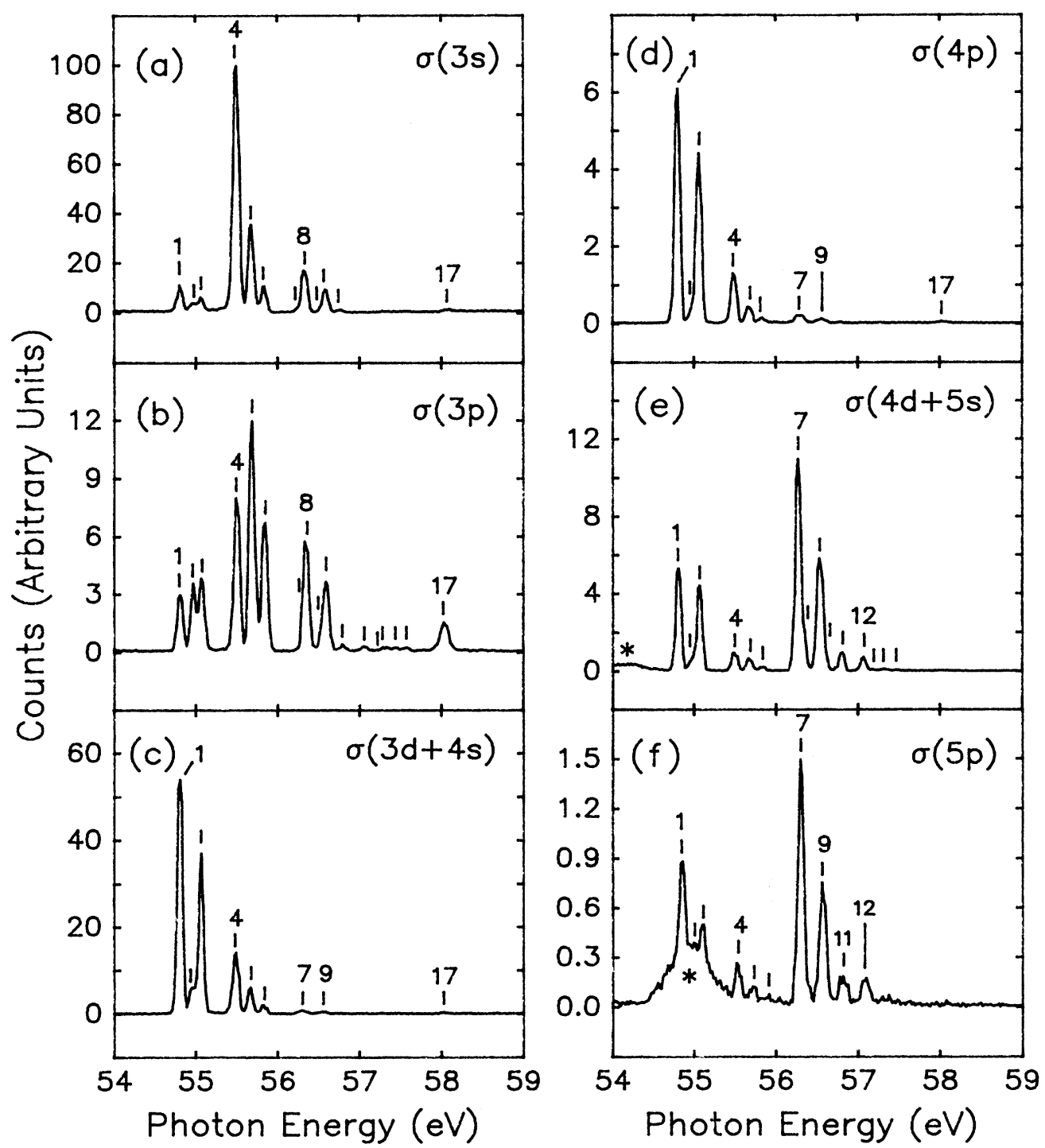

FIG. 2. Partial cross sections of the $3 s$ line and various correlation satellites through the $2 p \rightarrow n s(d)$ resonances as recorded by CIS scans. The energy scale is fixed at the $2 p_{3 / 2} \rightarrow 3 d$ resonance (4) at $55.49 \mathrm{eV}$ as taken from Ref. 10 . Resonance energies and assignments are given in Table I. All CIS spectra were taken with $20 \mathrm{meV} / \mathrm{step}$ at a resolution of $0.30 \AA$ ( $\cong 0.08 \mathrm{eV})$. The feature marked with an asterisk is an Auger interloper as described in the text.

partial cross sections of the $3 s$ line and various correlation satellites, are shown in Fig. 2. Table I gives the resonance energies and assignments of the excited states of the neutral which are relevant to this work. The resonance energies which we report were determined by using resonance 4 in Fig. 2(a) as a reference. The assignments and reference energy are taken from the photoabsorption work of Ederer, Lucatorto, and Mehlman. ${ }^{10}$ The scale on the left-hand side of Fig. 2 is in arbitrary units and is the actual scale by which the CIS and PES spectra were normalized with respect to each other. The ordinate of this figure can be converted to an absolute scale in $\mathrm{Mb}$ by multiplication with the factor $0.24(2)$. This conversion factor was determined by normalizing the $3 \mathrm{~s}$ cross section
[Fig. 2(a)] in the region from 54 to $54.78 \mathrm{eV}$ (assumed to be flat) to the theoretical value of $0.2 \mathrm{Mb}$ as reported by Altun ${ }^{12}$ (his Fig. 5).

A few comments and observations are in order regarding Fig. 2(a). Although a direct comparison with the many-body perturbation theory (MBPT) calculation of Altun ${ }^{12}$ for the $3 s$ cross section through the $2 p \rightarrow n s(d)$ resonances is somewhat difficult because his calculations have not been convoluted with a monochromator bandpass of $0.30 \AA$, we note the following. The theoretical calculation correctly predicts that the strongest resonance will be associated with the $2 p^{5} 3 s^{2} 3 d$ configuration. However, the theoretical calculation gives a value of about 20:1 for the ratio of the peak height of the strong- 


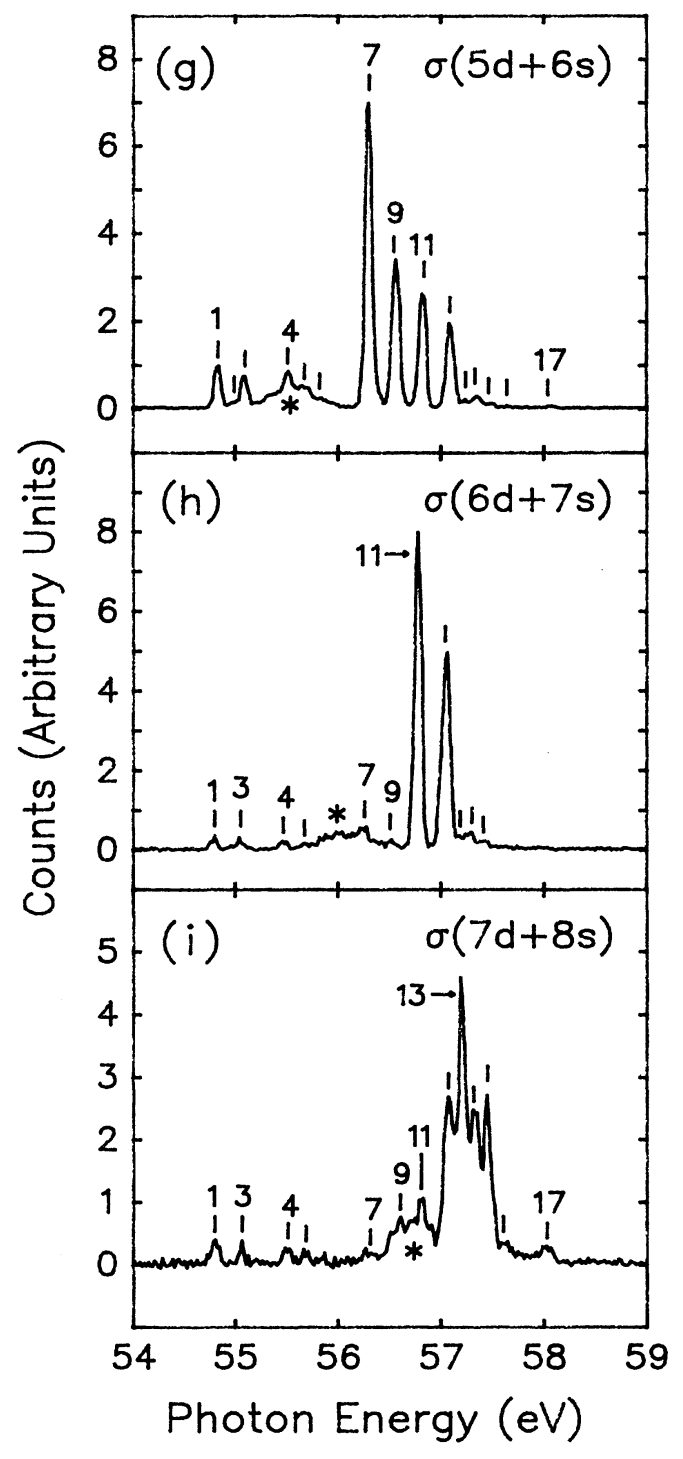

FIG. 2. (Continued).

est resonance $\left[2 p^{5} 3 d\left({ }^{3} D\right) 3 s^{2}\right]$ to the continuum. We obtain a ratio of about 120:1. Since a convolution of the theoretical cross section with a $0.30-\AA$ bandpass would only broaden the resonance and reduce its peak intensity, leaving the integrated intensity the same, we conclude that the theoretical calculation has seriously underestimated the strength of these resonances.

The cross sections of Fig. 2 clearly indicate that the higher the binding energy of the correlation satellite, the more strongly enhanced it becomes at the higher autoionizing resonances. It is also clear that in most cases, the correlation satellites are strongest at resonance energies for which the excited electron of the neutral has the same angular momentum as the correlation satellite and the same principal quantum number $n$ or $n^{\prime}=n \pm 1$. This is clearly the case for the excitations shown in Figs. 2(c), $2(\mathrm{e}), 2(\mathrm{~g}), 2(\mathrm{~h})$, and $2(\mathrm{i})$. This is an indication that when
TABLE I. Mg I resonance energies and their assignments. Numbers in parentheses denote error.

\begin{tabular}{cccc}
\hline \hline & \multicolumn{2}{c}{ Level energy (eV) } & \\
\cline { 2 - 3 } Resonance & This work & Ref. 10 & Assignment ${ }^{\mathrm{a}, \mathrm{b}}$ \\
\hline 1 & $54.81(3)$ & $54.802(5)$ & $3 s^{2}\left({ }^{2} P_{3 / 2}\right) 4 s$ \\
2 & $54.96(3)$ & $54.963(5)$ & {$\left[3 s\left(3 p^{2}{ }^{3} P\right)^{4} P\right]^{3} P$} \\
3 & $55.07(3)$ & $55.065(5)$ & $3 s^{2}\left({ }^{2} P_{1 / 2}\right) 4 s$ \\
4 & $55.49^{\mathrm{c}}$ & $55.491(5)$ & $3 s^{2}\left({ }^{2} P_{3 / 2}\right) 3 d$ \\
5 & $55.68(3)$ & $55.679(5)$ & {$\left[3 s\left(3 p^{2}{ }^{1} D\right)^{2} D\right]^{1} P$} \\
6 & $55.83(3)$ & $55.839(5)$ & $3 s^{2}\left({ }^{2} P_{1 / 2}\right) 3 d$ \\
7 & $56.30(3)$ & $56.277(5)$ & $3 s^{2}\left({ }^{2} P_{3 / 2}\right) 5 s$ \\
8 & $56.35(3)$ & $56.357(5)$ & $3 s^{2}\left({ }^{2} P_{3 / 2}\right) 4 d$ \\
9 & $56.56(3)$ & $56.544(5)$ & $3 s^{2}\left({ }^{2} P_{1 / 2}\right) 5 s$ \\
10 & $56.60(3)$ & $56.604(5)$ & $3 s^{2}\left({ }^{2} P_{1 / 2}\right) 4 d$ \\
11 & $56.78(3)$ & $56.777(5)$ & $3 s^{2}\left({ }^{2} P_{3 / 2}\right) 6 s$ \\
12 & $57.07(3)$ & $57.081(5)$ & $3 s^{2}\left({ }^{2} P_{3 / 2}\right) 6 d$ \\
& & & $3 s^{2}\left({ }^{2} P_{1 / 2}\right) 5 d$ \\
13 & $57.21(3)$ & $57.215(5)$ & $3 s^{2}\left({ }^{2} P_{3 / 2}\right) 7 d$ \\
14 & $57.34(3)$ & $57.347(5)$ & $3 s^{2}\left({ }^{2} P_{1 / 2}\right) 6 d$ \\
15 & $57.45(3)$ & $57.456(5)$ & $3 s^{2}\left({ }^{2} P_{1 / 2}\right) 8 s$ \\
16 & $57.63(3)$ & $57.634(5)$ & $3 s^{2}\left({ }^{2} P_{1 / 2}\right) 9 d$ \\
17 & $58.04(3)$ & $58.067(5)$ & {$\left[3 s\left(3 p^{2}{ }^{1} S\right)^{2} S\right]^{1} P$} \\
\hline \hline
\end{tabular}

${ }^{\text {a }}$ These assignments are taken from Ref. 10.

${ }^{\mathrm{b}}$ The notation used here only includes those electrons outside the $1 s^{2} 2 s^{2} 2 p^{5}$ core.

${ }^{\mathrm{c}}$ Reference energy.

the decay of an excited state of the neutral takes place via autoionization, the excited electron tends to remain a spectator, although shakeup and shakedown to adjacent $n$ levels can be quite strong. A more detailed discussion of this behavior will be given when we examine the PES results. We also observe that the cross section for the $4 p$ electron looks remarkably like that of the $3 d+4 s$ [Fig. $2(\mathrm{c})]$, while that of the $5 p$ looks remarkably like that of the $4 d+5 s$ [Fig. 2(e)]. This is somewhat surprising in view of the fact that the $3 p$ cross section looks markedly different from that of the $3 s$.

Finally, one will notice that in all the spectra beginning with Fig. 2(e) and continuing through Fig. 2(i), a broad, featureless structure can be seen tracking through the various cross sections at different photon energies. This feature has been marked with an asterisk in the spectra of Fig. 2. We have determined that this arises from an $L_{2,3}-M_{1} M_{1}$ Auger interloper which results from the creation of a $2 p$ hole by second-order light, despite the use of an Al filter to block out higher order radiation.

The sum of all the panels in Fig. 2 should closely reproduce the photoabsorption spectrum of Ref. 10 below the $2 p_{3 / 2}$ threshold. The result of this summation is shown in Fig. 3. We observe the same spectrum as seen by Ederer, Lucatorto, and Mehlman, ${ }^{10}$ although we cannot resolve the higher members of the $n d$ states from the $(n+1) s$ states for $n \geq 4$. The primary differences between our total cross section and the photoabsorption spectrum can be ascribed to the following three reasons: lack of several channels, differences in resolution, and 
differences in recording methods. We have not measured the partial cross sections for the $n p$ states for $n \geq 6$ and the $n s(d)$ states for $n \geq 9(8)$. However, we do not expect these states to contribute much to the total cross section. Probably the most significant difference between our work and Ref. 10 is due to resolution, $0.30(2) \AA$ in our case compared to $0.06 \AA$ in the photoabsorption work. In our measurement the broad bandpass will considerably reduce the peak height of the higher energy, more narrow resonances. Finally, we attribute remaining discrepancies to the differences in recording techniques, noting that the counting technique we use is superior to photographic recording in both linearity and dynamic range. Despite the limitations of the comparison between our total cross section and the absorption spectrum, there is, nevertheless, satisfactory agreement.

The experimental results for the low-resolution PES spectra $[\mathrm{FWHM}=0.38(2) \mathrm{eV}]$ following background subtraction, normalization as discussed previously, ${ }^{23}$ and a conversion to a binding energy scale are shown in Fig. 4. As a reference we have taken the binding energy of the $3 \mathrm{~s}$ photoline to be $7.644 \mathrm{eV} .^{24}$ In order to better resolve the satellite structure in the PES spectra recorded at the higher resonance photon energies, an additional series of high-resolution PES spectra $[\mathrm{FWHM}=0.20(2) \mathrm{eV}]$ were taken covering the region of the higher binding energy satellites only. These spectra (following background subtraction, normalization with respect to the low-resolution PES spectra, and conversion to a binding energy scale) are shown in Fig. 5. Normalization between the lowresolution PES spectra and the high-resolution PES spectra was accomplished by setting the integrated counts for all features in the high-resolution spectra equal to the integrated counts for the same features in the lowresolution spectra. The binding energies of the $3 \mathrm{~s}$ photoline and the observed correlation satellites are given in

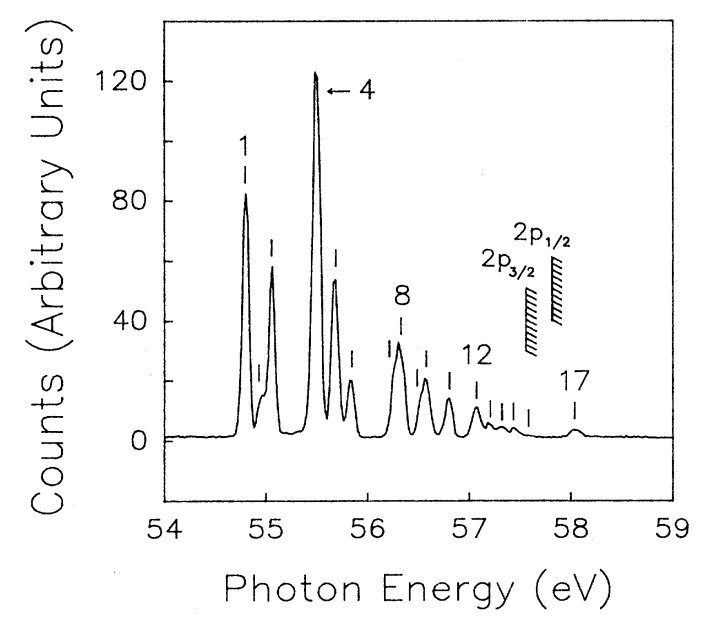

FIG. 3. Cross section of $\mathrm{Mg}$ through the $2 p \rightarrow n s(d)$ resonances. This figure is a result of adding all the panels in Fig. 2 together. Resonance energies and assignments are given in Table I. The positions of the $2 p_{3 / 2}$ and $2 p_{1 / 2}$ thresholds at $57.545(5)$ and $57.814(5) \mathrm{eV}$, respectively, are also indicated in the figure.
Table II, in addition to a comparison with optical data from which the assignments are taken. It should be noted that in all cases, except for $n=3,{ }^{25}$ we were unable to experimentally resolve the $n d$ from the $(n+1) s$ satellites.

Branching ratios for the decay of the various excited states of the neutral into the ground state of the ion and its numerous excited states are given in Table III. These measured values are true branching ratios defined by $B_{i}=\Gamma_{i} / \Gamma$ as opposed to relative intensities. Here $B_{i}$ is the branching ratio for the $i$ th decay channel of the excited state. $\Gamma_{i}$ is the partial width of the $i$ th decay channel, and $\Gamma$ is the sum of all the partial widths given by $\Gamma=\sum_{i} \Gamma_{i}$. Experimentally, the integrated intensity of a given feature in a PES spectrum $I_{i}$ is directly proportional to the partial width of that feature, hence, $B_{i}=I_{i} / \sum_{i} I_{i}$. Since we are interested in true branching ratios, we must be careful to subtract out any contribution to the integrated intensity of a spectral feature which might arise from direct photoionization. This is especially true for the most highly excited states, Figs. 4(j), 4(k), and $4(1)$, where, for example, the main $3 s$ photoline is nearly or completely due to direct photoionization.

An off-resonance spectrum taken at $80 \mathrm{eV}$ is shown in Fig. 4(a) for comparison with the on-resonance spectra. Because the cross section off resonance was so small, it was necessary to record this spectrum at a lower resolution $[F W H M=0.54(1)]$ than the other PES spectra in order to obtain a reasonable counting rate. The most striking feature of this spectrum is how small the cross sections of its features are compared to those of the other spectra. Even at the weakest resonance [Fig. 4(k)], the strongest feature is roughly eight times larger than the off-resonance $3 s$ cross section. This indicates very dramatically how weak the direct process of photoionization is compared to the indirect process of autoionization through the $2 p \rightarrow n s(d)$ resonances. This is also quite clear in the CIS spectra. The only appreciable correlation satellites which appear off resonance are the $3 p$ and $4 s+3 d$ satellites, and perhaps some $4 p$, which arise strictly from initial-state configuration interaction (ISCI). The relative intensities between the main line and its satellites due to ISCI are $I(3 p) / I(3 s)=0.133(6)$ and $I(4 s+3 d) / I(3 s)=0.077(5)$, where the $I(4 s+3 d)$ intensity may include a small amount of the $4 p$ satellite.

\section{B. Resonance 1: the $2 p_{3 / 2} \rightarrow 4 s$ excitation, $h v=54.81(3) \mathrm{eV}$}

The spectrum of the $3 s$ line and its correlation satellites at the $2 p_{3 / 2} \rightarrow 4 s$ excitation is shown in Fig. 4(b). One immediately sees a very dramatic difference from the offresonance spectrum of Fig. 4(a). The most intense peak in the spectrum is the $4 s$ satellite ${ }^{25}$ which is $5.3(3)$ times stronger than the $3 s$ line. In addition, we now observe three additional satellites which were not even visible in the off-resonance spectrum: the $4 p, 5 s, 26$ and the $6 s^{26}$ satellites. The strength of the $4 s$ satellite is a direct indication that the excited $4 s$ electron in the neutral likes to remain as a spectator during the autoionizing decay, in accordance with the spectator model. We also note that the presence of the $3 p$ and $4 p$ satellites is direct evidence for configuration mixing in the excited state of the neu- 

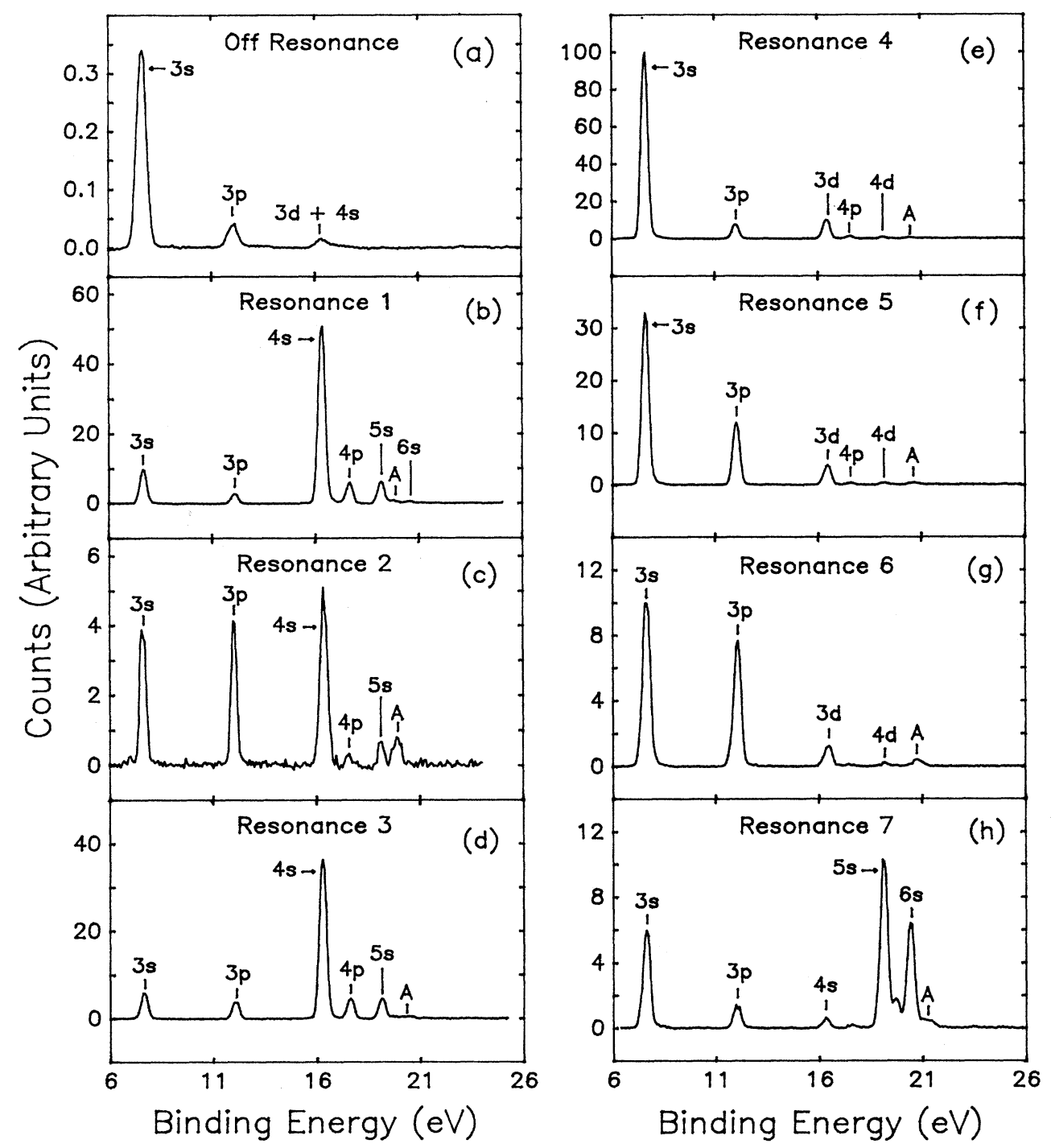

FIG. 4. PES electron spectra $[\mathrm{FWHM}=0.38(2) \mathrm{eV}]$ of atomic $\mathrm{Mg}$ at 12 different photon energies: (a) off resonance at $h v \cong 80 \mathrm{eV}$, (b) -(1) at various resonance energies as indicated in Table I. All spectra have the background subtracted and are normalized as indicated in the text. Assignments are based on the data of Ref. 24. The letter $A$ indicates the position of the $L_{2,3}-M_{1} M_{1}$ Auger lines, which arise from second-order radiation except in the case of Fig. 4(1). Each spectrum contains 256 points.

tral. These satellites cannot originate from the shakeup or shakedown of the $4 s$ electron because the overlap matrix elements for such processes vanish due to the orthogonality of the angular part of the wave functions. Finally, we note that the feature marked with an $A$ in the spectrum is the $L_{2,3}-M_{1} M_{1}$ Auger line which is a consequence of second-order radiation from the monochromator.

\section{Resonance 2: the $2 p \rightarrow 3 s 3 p^{2}\left({ }^{3} P\right)$ transition, $h v=54.96(3) \mathrm{eV}$}

The spectrum for this transition is shown in Fig. 4(c). As in the previous case, one observes a very strong enhancement of the correlation satellites, with the $3 p$ and
$4 s$ lines somewhat stronger than the $3 s$. The most obvious difference between this spectrum and the preceding spectrum is the relative strength of the $3 p$ line compared to both the main $3 s$ line and the $4 s$ line. The enhancement of the $3 p$ line with respect to the $3 s$ line is not surprising considering the configuration of the excited state, and suggests that one of the $3 p$ electrons frequently participates in the decay of this excited state. Since, however, one or both of the $3 p$ electrons must participate in the decay process, we cannot really speak of a spectator transition with respect to the $3 p$ electrons. However, we can speak of spectator transitions with respect to the $3 \mathrm{~s}$ electron. In this way we can understand the rather surprising strength of the $4 s$ line considering the configuration of the excited neutral. This line can arise 


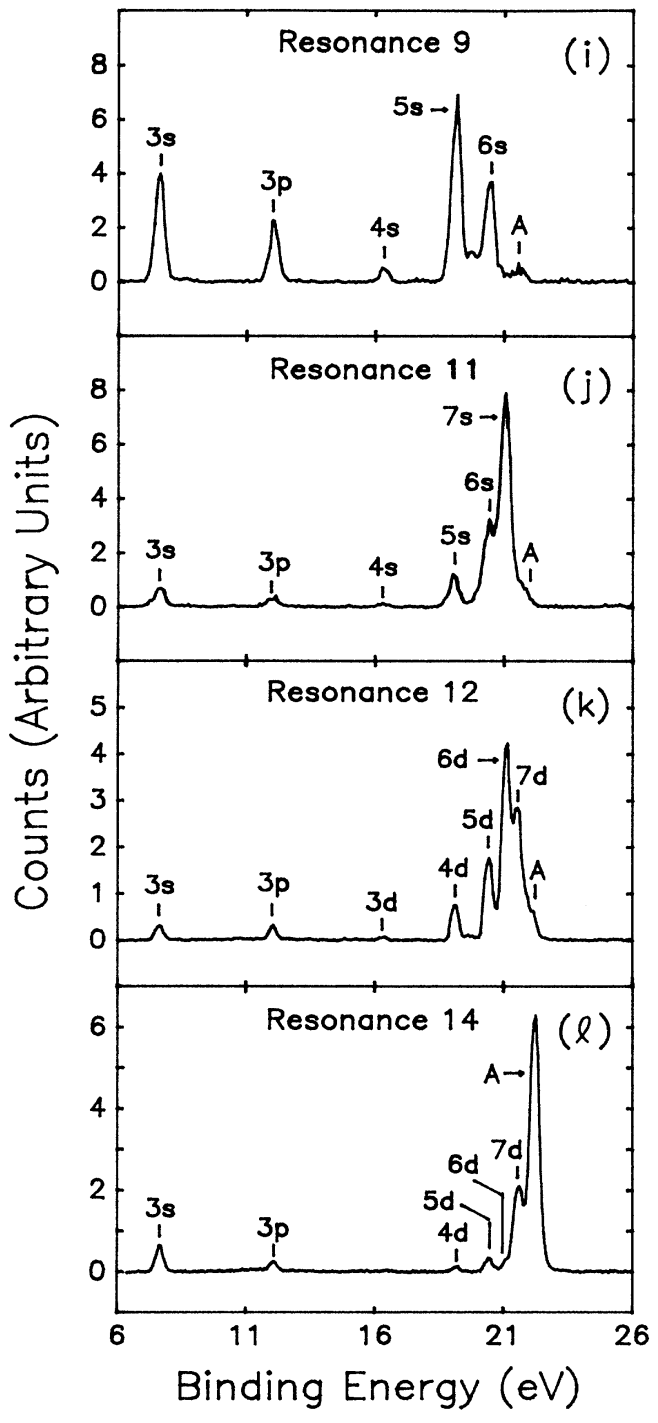

FIG. 4. (Continued).

either from initial excited state configuration mixing of the $3 p^{2} 4 s$ configuration, or, more likely, from the shakeup of the $3 s$ electron into the $4 s$ level. In fact, shakeup of the $3 s$ electron into the $5 s$ state is the most reasonable explanation for the appearance of the $5 s$ satellite. When viewed in this manner, we see that the spectator model qualitatively explains the decay dynamics of this resonance excitation.

\section{Resonance 3: the $2 p_{1 / 2} \rightarrow 4 s$ excitation, $h v=55.07(3) \mathrm{eV}$}

The spectrum for this transition is shown in Fig. 4(d). This decay looks virtually identical to that of Fig. 4(b), indicating that the total angular momentum of the core hole, whether $\frac{1}{2}$ or $\frac{3}{2}$, has little influence on the decay dynamics. There are, nevertheless, some slight differences. Most notable is the increased relative intensity of the $3 p$ satellite with respect to all other features in the spectrum.
TABLE II. Mg II $3 s$ and correlation satellite binding energies. Numbers in parentheses denote error.

\begin{tabular}{ccc}
\hline & \multicolumn{2}{c}{ Binding energy (eV) } \\
\cline { 2 - 3 } Level & This work & Ref. 24 \\
\hline $3 s$ & 7.644 & $7.644^{\mathrm{a}}$ \\
$3 p$ & $12.06(4)$ & 12.072 \\
$4 s$ & $16.32(4)$ & 16.299 \\
$3 d$ & $16.48(4)$ & 16.508 \\
$4 p$ & $17.61(4)$ & 17.642 \\
$5 s$ & $19.15(4)$ & 19.148 \\
$4 d$ & $19.15(4)$ & 19.213 \\
$5 p$ & $19.73(4)$ & 19.728 \\
$6 s$ & $20.47(4)$ & 20.439 \\
$5 d$ & $20.47(4)$ & 20.467 \\
$6 p$ & $20.77(4)$ & 20.740 \\
$7 s$ & $21.11(4)$ & 21.132 \\
$6 d$ & $21.11(4)$ & 21.146 \\
$7 p$ & $21.32(6)$ & $21.303^{\mathrm{b}}$ \\
$8 s$ & $21.57(4)$ & 21.546 \\
$7 d$ & $21.57(4)$ & 21.555 \\
\hline
\end{tabular}

${ }^{a}$ Reference energy.

${ }^{\mathrm{b}}$ This number is a result of a quantum-defect fit of the $4 p, 5 p$, and $6 p$ states whose energies are taken from Ref. 24.

In addition, we find that the branching ratio for the decay of the $4 s$ excited state into the ground state of the ion is smaller here, $B=9.2(4)$, than at the $2 p_{3 / 2} \rightarrow 4 s$ excitation [Fig. 4(b)] where $B=12.6(4)$, indicating that the participator decay is somewhat weaker in this instance.

\section{E. Resonance 4: the $2 p_{3 / 2} \rightarrow 3 d$ excitation, $h v=55.49 \mathrm{eV}$}

The spectrum for this transition is shown in Fig. 4(e). This decay looks remarkably like the off-resonance decay of Fig. 4(a), although the cross section of the $3 s$ line and the accompanying correlation satellites are approximately two orders of magnitude larger. Here we observe strikingly different behavior from the previous spectra. For the first time we see that participator decay dominates over spectator decay, indicating a complete breakdown of the spectator model. This behavior is not altogether surprising. Such a breakdown of the spectator model has been observed in the decay of the Ca $2 p^{5} 3 s^{2} 3 p^{6} 4 s^{2} 3 d$ photoexcited state. ${ }^{27}$ This breakdown was ascribed to the collapse of the $3 d$ wave function into a corelike orbital with an energy close to that of the $4 s$ orbital. It is reasonable to assume that a similar situation might also occur in this instance. If the excited $3 d$ electron orbital of magnesium were to collapse in the presence of the $2 p$ core hole, then there would be significantly more overlap between the vacant $2 p$ orbital and excited $3 d$ orbital. This increased overlap would greatly enhance the probability for the excited $3 d$ electron to participate in the decay of the $2 p$ hole. Such a collapse would be a strong indication of configuration interaction in the excited neutral, especially $3 s^{2} \leftrightarrow 3 d^{2}$, where it is well known that configuration interaction leads to a contraction of the single configuration orbital. Although we can qualitatively as- 
cribe the observed behavior at this resonance as resulting from the collapse of the $3 d$ orbital, detailed calculations are necessary to validate such a conjecture. As in the case of the $2 p \rightarrow 4 s$ excitations, we again note that the presence of the $3 p$ and $4 p$ satellites is direct evidence of configuration mixing in the excited state of the neutral.

\section{F. Resonance 5: the $2 p \rightarrow 3 s 3 p^{2}\left({ }^{1} P\right)$ excitation, $h v=55.68(3) \mathrm{eV}$}

The spectrum for this transition is shown in Fig. 4(f). The appearance of this spectrum is somewhat surprising. One might have expected that this spectrum would look

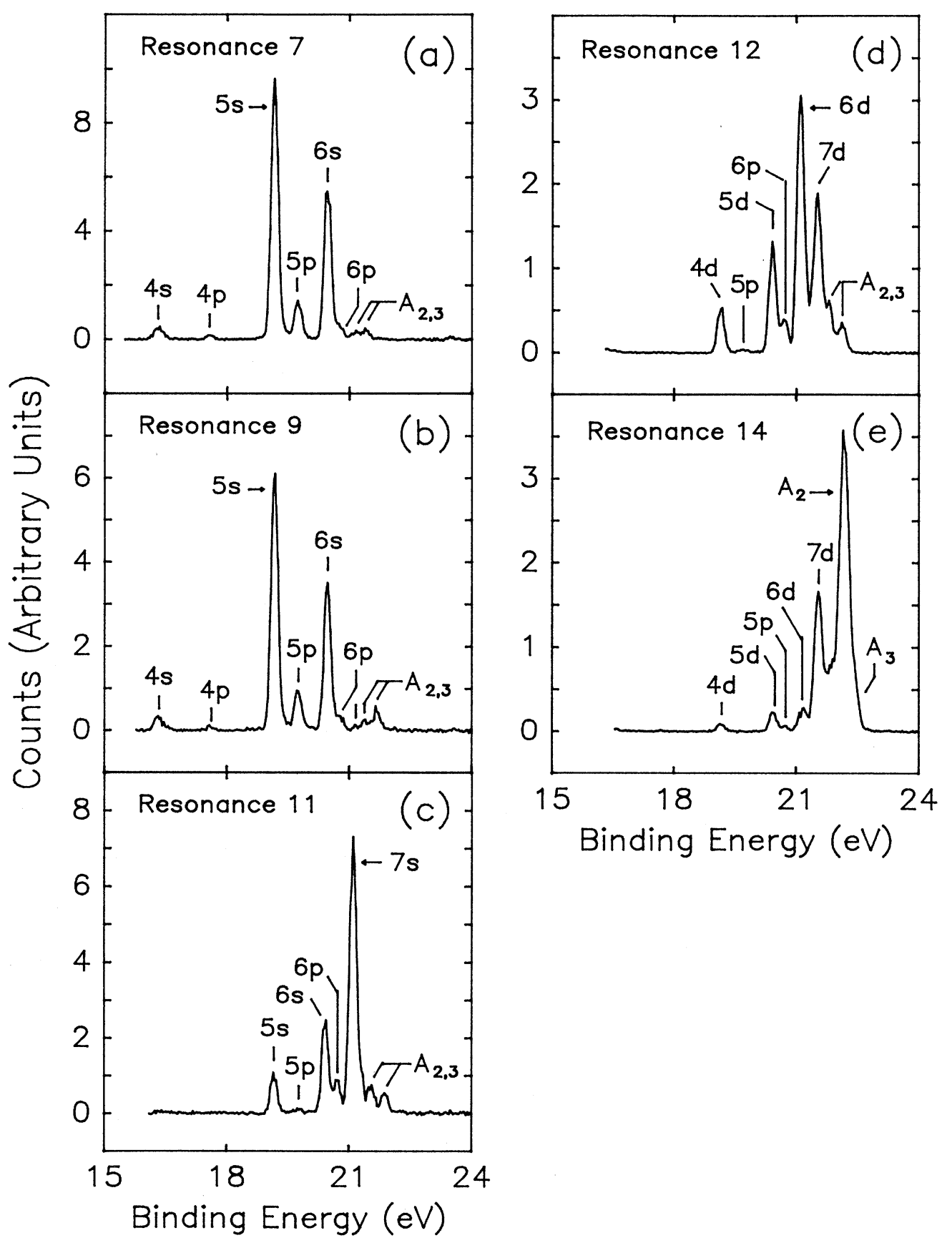

FIG. 5. High-resolution PES electron spectra $[\mathrm{FWHM}=0.20(2) \mathrm{eV}]$ of the higher binding energy satellites of atomic $\mathrm{Mg}$ at five different resonance energies as indicated in Table $I$. All spectra have the background subtracted and are normalized as indicated in the text. Assignments are based on the data of Ref. 24. The letters $A_{2}$ and $A_{3}$ indicate the positions of the $L_{2}-M_{1} M_{1}$ and the $L_{3}$ $M_{1} M_{1}$ Auger lines, respectively, which arise from second-order radiation except in the case of Fig. 5(e). Each spectrum contains 256 points. 


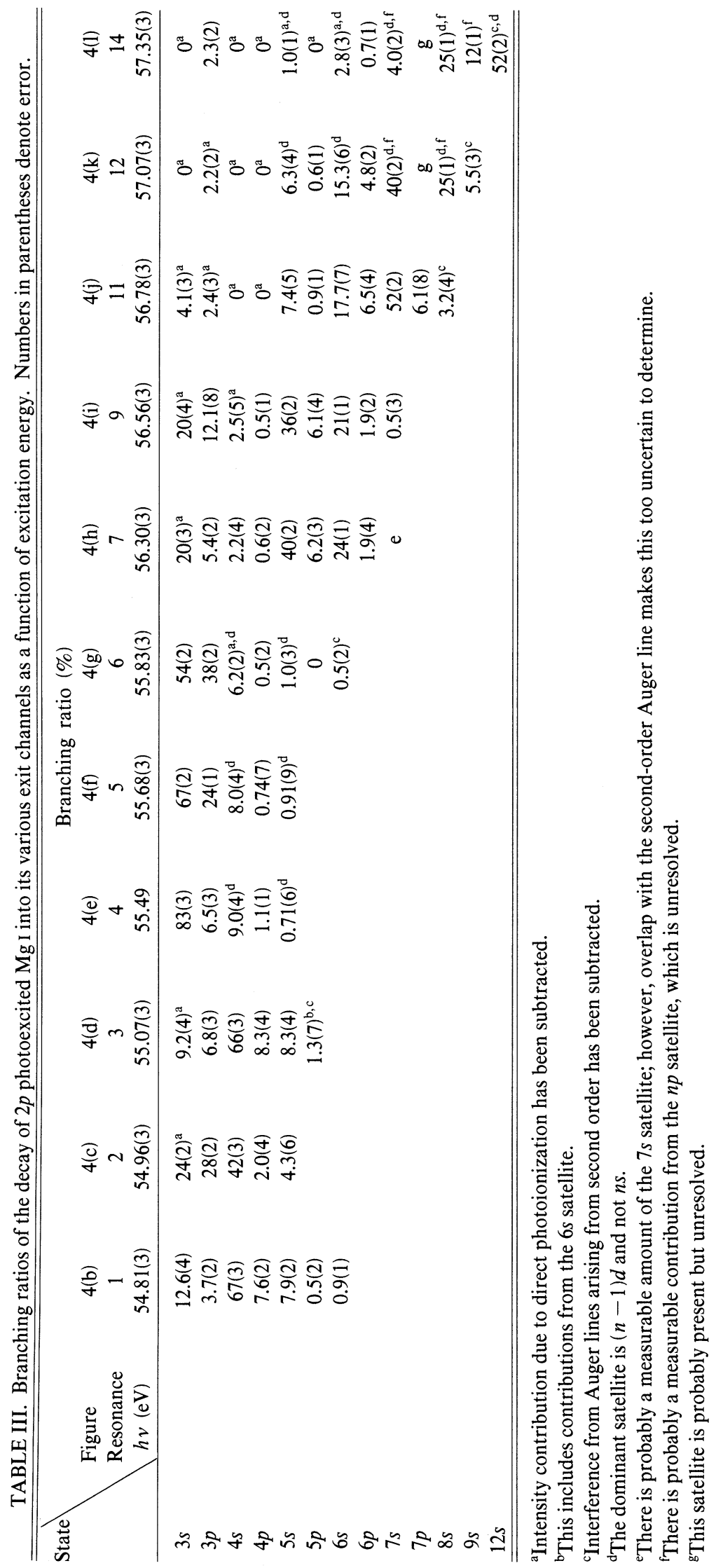


much more like that in Fig. 4(c), which, aside from the final term value, has the same configuration. Instead, it looks very similar to that of Fig. 4(e), with the major difference being the relative increase in intensity of the $3 p$ satellite with respect to all other features in the spectrum. This is in line with the enhancement of the $3 p$ satellite in Fig. 4(c) and can be explained in a similar manner. What cannot be explained is the complete absence of the $4 \mathrm{~s}$ line. ${ }^{25}$ We note that production of the ${ }^{3} P$ state in Fig. $4(\mathrm{c})$ requires a spin flip, and that the geneology of the coupling scheme is very different from the present case (see Table I). One consequence of this is the weakness of transition 2 compared to the present one. With respect to the decay process, the production of the $4 \mathrm{~s}$ in one case and not in the other suggests that spin flip might play an important role in shakeup. In addition to the $3 d$ satellite, we also observe a $4 d$ satellite. $^{25}$ Again, these satellites cannot arise from shakeup of the $3 s$ electron to the $4 d$ or $5 d$ levels because of orthogonality of the angular portion of the wave functions. Hence, these satellites can only be a result of ISCI in the excited neutral.

\section{G. Resonance 6: the $2 p_{1 / 2} \rightarrow 3 d$ excitation, $h v=55.83(3) \mathrm{eV}$}

The spectrum for this transition is shown in Fig. 4(g). Except for the increased relative intensity of the $3 p$ line with respect to all other features, this spectrum is not too different from that of Fig. 4(e). We note for example, that the relative intensity between the $3 d$ and $3 s$ lines and the $4 d$ and $3 s$ lines is roughly the same here as in Fig. 4(e). As in Fig. 4(e), the $3 d$ participator transition is the strongest decay channel, presumably due to the collapse of the $3 d$ wave function. This could explain the sensitivity of the $2 p \rightarrow 3 d$ excitations to the angular momentum of the core hole, which is absent in the case of the $2 p \rightarrow 4 s$ excitations. Thus, the primary difference between the decay of the $2 p_{3 / 2}^{-1} 3 s^{2} 3 d$ excited state [Fig. 4(e)] and this state is not that there is stronger mixing of the $3 d 3 p^{2}$ configuration in this state, but rather a weaker probability for participator decay in this state. This is clear from the CIS spectrum of Fig. 2(b) where the strength of the $3 p$ cross section is nearly equal at resonances 4 and 6 . In fact, the $3 s$ line is approximately one order of magnitude stronger at the $2 p_{3 / 2} \rightarrow 3 d$ transition than it is here.

\section{H. Resonance 7: the $2 p_{3 / 2} \rightarrow 5 s$ excitation, $h v=56.30(3) \mathrm{eV}$}

The spectrum for this transition is shown in Fig. 4(h). In Fig. 5(a) the higher members of the $3 s$ correlation satellites are shown at high resolution. We now observe the presence of new satellite lines which were virtually nonexistent at lower excitation energies. Not only do we completely resolve the $5 p$ satellite in the high-resolution spectrum, but we also partially resolve the $6 p$ satellite from the $6 s$, and nearly resolve the $L_{2}-M_{1} M_{1}$ Auger line from the $L_{3}-M_{1} M_{1}$ Auger line. As observed for the $2 p \rightarrow 4 s$ excitations, we immediately see that the strongest autoionizing decay is that in which the excited $5 s$ electron remains a spectator, in accord with the spectator model. Furthermore, we see for the first time that shakeup of the excited electron is very pronounced. This is reflected by the relative intensity of the $6 s$ satellite compared to all other features in the spectrum. This behavior has been observed in the decay of the $1 s 2 s^{2} 3 p$ excited state of Be (Ref. 7) and in the decay of the $2 p$ core-excited states of Ar ( Ref. 19) and the $3 d$ core-excited states of $\mathrm{Kr}^{21}$ In fact, the probability that the $5 \mathrm{~s}$ electron will shake up to the $6 s$ level during decay is slightly more likely than the probability for it to participate during the decay (see Table III). We also note that the probability for shakedown of the $5 s$ electron to the $4 s$ state is quite weak, being roughly 11 times less likely than shakeup to the $6 s$ state.

\section{Resonance 9: the $2 p_{1 / 2} \rightarrow 5 s$ excitation, $h v=56.56(3) \mathrm{eV}$}

The spectrum for this transition is shown in Fig. 4(i) (low resolution) and in Fig. 5(b) (high resolution). In the high-resolution spectrum we now see evidence of the $7 \mathrm{~s}$ satellite which was obscured by the $L_{2}-M_{1} M_{1}$ Auger line in Fig. 5(a). Both of these spectra look remarkably similar to those in Figs. 4(h) and 5(a); the degree of similarity is consistent with that found between the $2 p_{3 / 2} \rightarrow 4 s$ and the $2 p_{1 / 2} \rightarrow 4 s$ excitations [Figs. $4(\mathrm{~b})$ and $4(\mathrm{~d})$, respectively]. This again indicates that in the case of $2 p \rightarrow n s$ excitations, the total angular momentum of the core hole plays a negligible role in the decay process. The primary difference between these spectra and those of Figs. 4(h) and $5(a)$ is the relative increase in the strength of the $3 p$ satellite relative to the main photoline and all other satellites in the spectrum. This is also consistent with regard to the $2 p \rightarrow 4 s$ transitions, for which the $3 p$ line was found to be stronger relative to all features in the spectrum recorded at the $2 p_{1 / 2} \rightarrow 4 s$ transition energy.

\section{J. Resonance 11: the $2 p_{3 / 2} \rightarrow 6 s$ excitation, $h v=56.78(3) \mathrm{eV}$}

The spectrum for this transition is shown in Fig. 4(j) (low resolution) and in Fig. 5(c) (high resolution). At this resonance energy we now see evidence of the $7 p$ satellite which forms a shoulder on the high binding energy side of the $7 s$ line. Furthermore, the apparent enhancement of the $L_{2}-M_{1} M_{1}$ line is most likely due to the $8 s$ satellite which at this photon energy has nearly the same kinetic energy as the Auger line. The appearance of this spectrum is quite different from those involving previous excitations of $2 p$ electrons to $n s$ states. First, the probability for a participator transition is much smaller. The contribution to the strength of the $3 s$ photopeak in this spectrum is due as much to direct photoionization as to autoionization. This is very apparent in the CIS spectrum of Fig. 2(a). Secondly, we see, for the first time, that shakeup of the excited electron during autoionization is substantially stronger than the spectator transition itself. This behavior was also observed following the decay of the $2 p_{3 / 2} \rightarrow 3 d$ excitation in Ar. ${ }^{19}$ In that article it was reported that shakeup of the $3 d$ electron to the $4 d$ state during autoionization was stronger than the spectator decay, although no quantitative analysis was given. A more quantitative analysis was carried out for $\mathrm{Kr},{ }^{21}$ where it was found that shakeup following the decay of the $3 d \rightarrow n p \quad(n=6,7,8)$ excitations was significantly more 
pronounced than the pure spectator transitions. Similar behavior has also been seen in $\mathrm{Be},{ }^{28}$ where it was found that shakeup of an excited $4 p$ electron to the $5 p$ level following autoionization of the $1 s 2 s^{2} 4 p$ state led to $I(5 p) / I(4 p)=1.6(7)$. From the branching ratios given in Table III we deduce that the relative intensity between the $7 s$ satellite and the $6 s$ satellite is $I(7 s) / I(6 s)=2.9(2)$. In addition, we also note that the relative probability for shakedown is much stronger here than in any previous spectrum. This is not so surprising, if one considers that the difference between adjacent wave functions for higher Rydberg states is not as marked as the difference between adjacent wave functions for the lower-lying states.

\section{K. Resonance 12: the $2 p_{3 / 2} \rightarrow 6 d, 2 p_{1 / 2} \rightarrow 5 d$ excitation, $h v=57.07(3) \mathrm{eV}$}

The spectrum for this transition is shown in Fig. 4(k) (low resolution) and in Fig. 5(d) (high resolution). In the high-resolution spectrum we completely resolve the $5 p$ satellite and much of the $6 p$ satellite, which is completely buried in the lower-resolution spectrum. These spectra are similar in many ways to the two previous spectra [Figs. 4(j) and 5(c)], showing marked population of the states adjacent to the spectator lines. The most noticeable difference is the presence of a new satellite, the $7 d$ (which would correspond to an $8 s$ line in the two previous spectra). Furthermore, we conclude on the basis of the CIS spectrum [Fig. 2(a)] that the entire strength of the $3 \mathrm{~s}$ photoline is due to direct photoionization. In addition, we can again resolve the two Auger lines. As before, we note the apparent enhancement of the $L_{2}-M_{1} M_{1}$ Auger line. This is due to a superposition of the Auger line and the $8 d$ satellite which at this photon energy has nearly the same kinetic energy as the Auger line.

A precise determination of the physics at this resonance energy is somewhat difficult because there are two degenerate excited states, and it is not possible to determine experimentally which excitation has the stronger cross section. However, if we assume that decay of both of these excited states proceeds in a manner similar to the previous resonance, we can then argue that the features present in this spectrum result equally from decay of both excited states. In the case of the $2 p_{3 / 2} \rightarrow 6 d$ transition, we would expect to see the dominant decay process to be the shakeup of the $6 d$ electron to the $7 d$ level, with a fairly strong spectator line and some shakedown of the $6 d$ to the $5 d$ level. In the case of the $2 p_{1 / 2} \rightarrow 5 d$ transition, we again expect the dominant decay process to involve shakeup of the $5 d$ electron to the $6 d$ state with some shakedown to the $4 d$ state and an appreciable amount of spectator line. If we then add these two processes, it is quite conceivable that one might reproduce the spectra shown in Figs. $4(\mathrm{k})$ and $5(\mathrm{~d})$. Although this is a plausible explanation for the behavior of these spectra, a detailed theoretical calculation is needed to truly elucidate the decay dynamics in this instance.

L. Resonance 14: the $2 p_{1 / 2} \rightarrow 6 d$ excitation, $h v=57.34(3) \mathrm{eV}$

The spectrum for this transition is shown in Fig. 4(1) (low resolution) and in Fig. 5(e) (high resolution). In the high-resolution spectrum we see evidence of shakeup to the $8 d$ level as well as the expected strong shakeup to the $7 d$ level. Somewhat surprising, however, is the relative weakness of the spectator transition and the relative strength of the $5 d$ and $4 d$ shakedown lines. Most unexpected is the terrific enhancement of the $L_{2}-M_{1} M_{1}$ Auger line below the $2 p_{1 / 2}$ threshold.

The appearance of an Auger line below its threshold is quite surprising, and one must be very careful to rule out any possibility that it arises from an experimental artifact. One possibility is that the bandpass of the incident radiation is broad enough to overlap with part of the $2 p_{1 / 2}$ threshold. At this resonance energy the bandpass of the monochromator is close to $0.08 \mathrm{eV}$. The difference in energy between this level and the $2 p_{1 / 2}$ threshold is $0.464 \mathrm{eV},{ }^{10}$ which is nearly six times greater than the bandpass. Although the $2 p_{1 / 2}$ threshold is not a step function, it is very unlikely that it would be spread out as far as $0.464 \mathrm{eV}$. In fact, according to Krause and Oliver, ${ }^{29}$ the width of the $2 p$ hole in $\mathrm{Mg}$ is approximately $0.001 \mathrm{eV}$. Furthermore, since we are much closer to the $2 p_{3 / 2}$ threshold at this excitation energy $(\cong 0.20 \mathrm{eV}$ below), we would also expect to see an enhancement of the $L_{3}-M_{1} M_{1}$ Auger line, which is not observed. Another possibility is improper normalization of this PES spectrum, indicating that all of the observed Auger line is a result of second-order light only. If this were in fact the case, we would again expect to see not only the $L_{2}-M_{1} M_{1}$ line in the high-resolution spectrum, but also the $L_{3}$ $M_{1} M_{1}$ line with roughly twice the intensity. Again, this is not observed. Furthermore the peak height of the $3 \mathrm{~s}$ line agrees very well with the off-resonance value determined from the CIS scan of Fig. 2(a).

To help clarify this unexpected result, we enlist the aid of two CFS scans carried out on the two Auger lines. In each CFS scan the Auger lines were tracked below both $2 p$ thresholds. The cross section of the $L_{2}-M_{1} M_{1}$ line as a function of photon energy is shown in Fig. 6(a), while the cross section of the $L_{3}-M_{1} M_{1}$ line as a function of photon energy is shown in Fig. 6(b). The $2 p_{1 / 2}$ and $2 p_{3 / 2}$ thresholds are indicated by the vertical lines in the spectra. The energy scale of these spectra was normalized to the value given in Ref. 10 for the window resonance near $61 \mathrm{eV}$. Normalization of these spectra and background subtraction were carried out in the manner discussed previously. The very strong feature in both spectra which peaks just above $58 \mathrm{eV}$ corresponds to the $2 p^{-1}\left[3 s\left(3 p^{2}{ }^{1} S\right)^{2} S\right]^{1} P$ excited state at $58.067(5)$ eV. ${ }^{10}$ The feature which lies below both the $2 p$ thresholds in Fig. 6(a) corresponds to a photon energy of $57.39(3) \mathrm{eV}$, in excellent agreement with the $2 p_{1 / 2} \rightarrow 6 d$ excitation energy determined from the CIS spectra. Important to note is that the CFS spectrum of the $L_{3}-M_{1} M_{1}$ line shows no enhancement at this photon energy, in complete agreement with the PES results. We therefore conclude that the apparent enhancement of the $L_{2}-M_{1} M_{1}$ Auger line below threshold is in fact a real phenomenon.

One possible explanation for this enhancement is the Auger resonant Raman process ${ }^{30}$ where the Auger diagram line has been observed below threshold. A primary 
requirement for the Auger resonant Raman process is for the bandwidth of the exciting radiation to be substantially smaller than the natural widths of the levels involved in the resonant process. However, the principal reason why the Auger diagram line was observed below threshold in Ref. 30 was due to the very broad nature of the initial core-hole state. The width of this state was such that photon energies which were technically below its threshold could still excite the core electron into the continuum. Subsequent decay of this core hole then led to the emission of an Auger line "below" threshold. As indicated above, the $2 p$ core-hole states of $\mathrm{Mg}$ are far too narrow for this effect to be possible.

We propose the following explanation as to the origin of the Auger enhancement. The difference in energy between the $\mathrm{Mg} 2 p_{1 / 2}^{-1} 3 s^{2} 6 d$ excited state and the $\mathrm{Mg}^{+}$ $2 p^{6} 6 d$ state is $36.12 \mathrm{eV}$, while the energy difference between the same excited state and the $\mathrm{Mg}^{2+} 2 p^{6}$ state is $34.67 \mathrm{eV}$. The kinetic energy of the $L_{2}-M_{1} M_{1}$ line is $35.14 \mathrm{eV},{ }^{31}$ which lies between the two values given

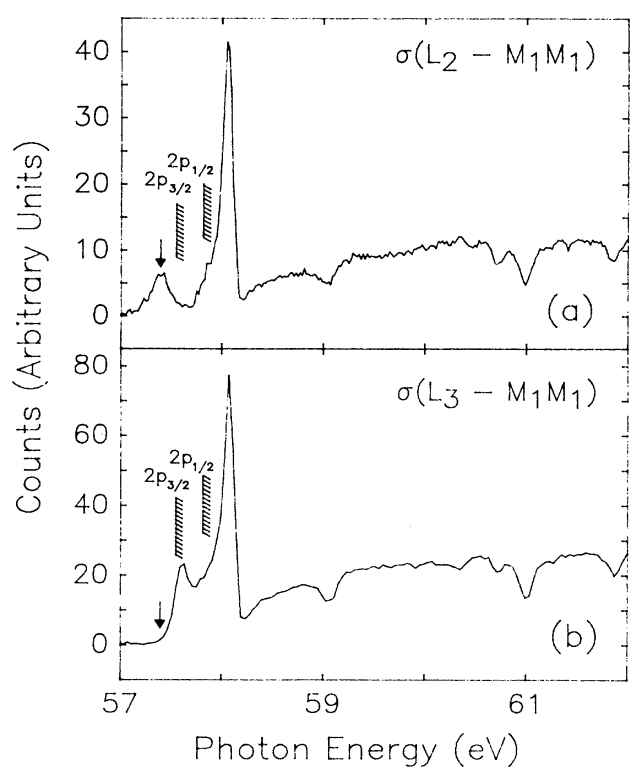

FIG. 6. Partial cross sections of the $L_{2}-M_{1} M_{1}$ Auger line (a) and the $L_{3}-M_{1} M_{1}$ Auger line (b) from $57.0-62.0 \mathrm{eV}$. The energy scale is fixed at the window resonance at $60.989(5) \mathrm{eV}$ as taken from Ref. 10. The $2 p_{3 / 2}$ and the $2 p_{1 / 2}$ thresholds are indicated by the two vertical lines at $57.545(5)$ and $57.814(5) \mathrm{eV}$, respectively. These spectra were taken with a resolution of $0.30 \AA$ ( $\cong 0.08 \mathrm{eV}$ ). The $L_{2}-M_{1} M_{1}$ spectrum was recorded with 20 $\mathrm{meV} / \mathrm{step}$ while the $L_{3}-M_{1} M_{1}$ spectrum was recorded with 40 $\mathrm{meV} / \mathrm{step}$. The symmetric feature which appears below both $2 p$ thresholds in spectrum (a) at $57.39(3) \mathrm{eV}$, as indicated by the arrow, corresponds to the $2 p_{1 / 2} \rightarrow 6 d$ resonance. The very strong feature which appears in both spectra at $58.05(3) \mathrm{eV}$ corresponds to the $2 p \rightarrow\left[3 s\left(3 p^{2}{ }^{1} S\right)^{2} S\right]^{1} P_{1}$ resonance. The spectrum of Fig. 6(a) was normalized by setting the peak height of the $2 p_{1 / 2}-6 d$ transition equal to the peak height of the $L_{2}-M_{1} M_{1}$ Auger line in Fig. 4(1). A relative intensity of 2:1 was assumed between Figs. 6(b) and 6(a). above. This strongly suggests that what we are seeing is the decay of the $\mathrm{Mg} 2 p_{1 / 2}^{-1} 3 s^{2} 6 d$ excited state into some $\mathrm{Mg}^{+} 2 p^{6}$ nd state with the emission of an electron having the same kinetic energy as the Auger line, thereby causing an apparent enhancement of the Auger line below threshold. This same mechanism was used to explain the much smaller enhancement of the $L_{2}-M_{1} M_{1}$ Auger line in Figs. 5(c) and 5(d). Using quantum defect theory and the level energies of $\mathrm{Mg}$ II (Ref. 24) we calculated the binding energies of the $\mathrm{Mg}^{+} 2 p^{6} n d$ states from $n=9-12$. We found that the difference in energy between the $\mathrm{Mg}$ $2 p_{1 / 2}^{-1} 3 s^{2} 6 d$ excited state and the $\mathrm{Mg}^{+} 2 p^{6} 11 d$ state gives a kinetic energy of an emitted electron of $35.12 \mathrm{eV}$, experimentally indistinguishable from the Auger electron energy. The fact that there might exist an autoionizing electron with the same kinetic energy as an Auger electron is not surprising. What is surprising, however, is the intensity of this autoionizing line. Based on the shakeup trends in the spectra preceeding those at this resonance energy, we would have expected the $7 d$ satellite to be the strongest. Yet, this was not the case. It would seem as if the system wants to decay by emitting an electron of the same kinetic energy as the $L_{2}-M_{1} M_{1}$ Auger line even if that means substantial shakeup of the excited electron. Furthermore, it is clear from the CFS spectra of Fig. 6 that this behavior is unique to the $L_{2}-M_{1} M_{1}$ Auger line and not the $L_{3}-M_{1} M_{1}$ Auger line. Although we do not have a quantitative explanation for this effect, we can make the following physical argument. Because the decay of the core hole occurs so rapidly, it does not perceive that the high-lying Rydberg electron is still in the vicinity when the decay begins. Therefore, it produces an Auger electron exactly as if the excited Rydberg electron were free. As the Rydberg electron notices the alteration in the core, it is then captured into that orbital of the ion which satisfies the energy balance equation. Besides the obvious theoretical scrutiny, measurements involving $n d \geq 7 d$ states at much higher resolution and ion yield measurements of $\mathrm{Mg}^{+}$and $\mathrm{Mg}^{2+}$ are clearly desirable.

\section{SUMMARY}

The clear trend apparent in nearly all the spectra of Figs. 4 and 5 is the enhancement of the higher binding energy satellites over the main line as one excites the $2 p$ electron to higher and higher Rydberg states. This is also reflected in the CIS spectra. This is a definite indication that the dominant decay processes for these excited states are spectator transitions rather than participator transitions, which is in excellent agreement with the predictions of the spectator model. In fact, only for the excitation of the $2 p$ electrons to the $3 d$ state and for the excitation of the $2 p_{1 / 2} \rightarrow 6 d$ state does the spectator model break down, the former most likely resulting from the collapse of the $3 d$ wave function, and the latter due to an unknown threshold phenomenon. In addition, it is clear that shake processes become ever stronger as one excites to higher and higher Rydberg states, with shakeup eventually dominating over the pure spectator transition itself.

We can understand in a very qualitative sense why 
shakeup becomes so strong at the higher resonance energies. Following the spectator decay of an excited atom, the orbital of the excited atom will find that it has collapsed in going from the neutral to the ion. Because of this collapse, the excited electron may find that it overlaps more strongly with the $(n+1)$ th orbital of the ion than with the $n$th orbital of the ion. Hence, the excited electron will "automatically" go into the $(n+1)$ th orbital upon decay of the excited state. This picture has already been demonstrated in the case of $\mathrm{Ar}$ (Ref. 19) and $\mathrm{Kr}$ (Ref. 21) using the independent-particle shake model. We now confirm its validity for $\mathrm{Mg}$ as well.

In the case of $\mathrm{Mg}$, the principal matrix element governing the decay of the excited neutral via a spectator transition is

$$
\left\langle 2 p^{5} 3 s^{2} n s\left({ }^{2 s+1} L\right)\left|\sum_{i<j} 1 / r_{i j}\right| 2 p^{6} n s \varepsilon l\left({ }^{2 s+1} L\right)\right\rangle,
$$

which reduces to

$$
\left\langle 3 s(1) 3 s(2)\left({ }^{2 s+1} L\right)\left|1 / r_{12}\right| 2 p(1) \varepsilon l(2)\left({ }^{2 s+1} L\right)\right\rangle\left\langle n s^{\prime} \mid n s\right\rangle \text {, }
$$

where $\varepsilon$ represents the energy of the continuum electron and $l$ its angular momentum. The $\left|n s^{\prime}\right\rangle$ wave function represents the excited $n s$ electron in the presence of the $2 p$ hole, while the $|n s\rangle$ wave function represents the excited $n s$ electron in the presence of the $3 s$ holes. All other overlap matrix elements have been assumed to be equal to 1. The principal matrix element governing the decay of the excited neutral via a spectator transition involving shakeup or shakedown is

$$
\left\langle 2 p^{5} 3 s^{2} n s\left({ }^{2 s+1} L\right)\left|\sum_{i<j} 1 / r_{i j}\right| 2 p^{6}(n+\alpha) s \varepsilon^{\prime} l\left({ }^{2 s+1} L\right)\right\rangle,
$$

which reduces to

$$
\left\langle 3 s(1) 3 s(2)\left({ }^{2 s+1} L\right)\left|1 / r_{12}\right| 2 p(1) \varepsilon^{\prime} l(2)\left({ }^{2 s+1} L\right)\right\rangle\left\langle n s^{\prime} \mid(n+\alpha) s\right\rangle,
$$

where $\alpha$ is an integer greater than or equal to 1 for shakeup and less than or equal to negative 1 for shakedown. The square of the ratio of Eq. (2) to Eq. (1) gives the relative intensity between the spectator transition involving shakeup or shakedown and the spectator transition in the absence of shakeup or shakedown. If we assume that the two electron matrix elements in equations (1) and (2) are nearly the same (a reasonable approximation if the energy $\varepsilon^{\prime}$ is not too different from $\varepsilon$ ), we then find

$I((n+\alpha) s) / I(n s) \cong\left[\left\langle n s^{\prime} \mid(n+\alpha) s\right\rangle /\left\langle n s^{\prime} \mid n s\right\rangle\right]^{2}$.

Using only single-configuration wave functions obtained from the HF77 (Ref. 32) code, and using Eq. (3), we determined the relative intensity between the $(n+1) l$ $[(n-1) l]$ shakeup [shakedown] line and the $n l$ spectator line and between the $(n+2) l$ shakeup line and the $n l$ spectator line for four different resonance energies. The results are compared with our experimental values and given in Table IV. As can be seen from the table, the re- sults are quite good for the $s$ states, although in nearly all cases the theory overestimates the relative intensities. In the case of the $6 d$ state, however, the results are not as good. Here the theory even fails to correctly predict the observed experimental trend, which indicates substantial shakedown to the $n-1$ level and shakeup to the $n+1$ level of greater magnitude than shakeup to the $n+2$ level. This is most likely due to the use of singleconfiguration wave functions, where, in general, $d$ orbitals are known to be more highly correlated than $s$ orbitals. Nevertheless, we may still conclude that the important physics regarding these shake transitions is handled well by shake theory, which confirms the picture of a collapsing Rydberg orbital when going from the excited neutral to the excited ion. Finally we note that shake theory cannot possibly explain the shakeup of the $6 d$ electron to the $11 d$ state. According to shake theory the relative intensity between the $11 d$ state and the $6 d$ state is $3.25 \times 10^{-5}$ while experiment gives $13.0(8)$.

\begin{tabular}{|c|c|c|c|c|c|c|c|c|}
\hline \multirow[b]{2}{*}{ Resonance } & \multirow[b]{2}{*}{ Figure } & \multirow[b]{2}{*}{$n l$} & \multicolumn{2}{|c|}{$I((n-1) l) / I(n l)$} & \multicolumn{2}{|c|}{$I((n+1) l) / I(n l)$} & \multicolumn{2}{|c|}{$I((n+2) l) / I(n l)$} \\
\hline & & & Theor. & Expt. & Theor. & Expt. & Theor. & Expt. \\
\hline 1,3 & $4(\mathrm{~b}), 4(\mathrm{~d})$ & $4 s$ & & & 0.11 & $0.122(5)^{\mathrm{a}}$ & 0.001 & b \\
\hline 7,9 & $5(a), 5(b)$ & $5 s$ & 0.09 & $0.06(1)^{a}$ & 0.73 & $0.59(6)^{\mathrm{a}}$ & 0.002 & b \\
\hline 11 & $5(c)$ & $6 s$ & 0.75 & $0.42(3)$ & 4.19 & $2.9(2)$ & 0.26 & $0.18(2)$ \\
\hline 14 & $5(e)$ & $6 d$ & $0^{c}$ & $0.70(8)$ & 1.67 & $6.3(4)$ & 4.67 & $3.0(3)$ \\
\hline
\end{tabular}

TABLE IV. Experimental and theoretical relative intensities between the $(n+1) l[(n-1) l]$ shakeup [shakedown] line and the $n l$ spectator line and between $(n+2) l$ shakeup line and the $n l$ line at four different resonance energies. The theoretical results are calculated using Eq. (3). Numbers in parentheses denote error.

${ }^{\text {a }}$ This value is the result of averaging the $2 p_{1 / 2}$ and the $2 p_{3 / 2}$ channels.

${ }^{\mathrm{b}}$ This value is too small to be determined experimentally.

${ }^{\mathrm{c}}$ The actual value calculated is $1.3 \times 10^{-5}$. 


\section{ACKNOWLEDGMENTS}

We would like to extend our thanks and appreciation to R. Hodgekinson for assistance during the experimental portion of this work. This work was supported by the National Science Foundation (NSF) under Grant No.
PHY-8907286 and by the Division of Chemical Sciences, Office of Basic Energy Research, under Contract No. DE-AC05-850R21400 with Martin Marietta Energy Systems, Inc. The University of Wisconsin Synchrotron Radiation Center is operated under NSF Grant No. DMR8821625 .
${ }^{1}$ R. A. Rosenberg, M. G. White, G. Thornton, and D. A. Shirley, Phys. Rev. Lett. 43, 1384 (1979).

${ }^{2}$ H. Kobrin, R. A. Rosenberg, U. Becker, S. Southworth, C. M. Truesdale, D. W. Lindle, G. Thornton, M. G. White, E. D. Poliakoff, and D. A. Shirley, J. Phys. B 16, 4339 (1983).

${ }^{3}$ A. Yagishita, S. Aksela, Th. Prescher, M. Meyer, M. Richter, E. von Raven, and B. Sonntag, J. Phys. B 21, 945 (1988).

${ }^{4}$ M. G. Flemming, S. B. Whitfield, C. D. Caldwell, J. JimenezMier, P. van der Meulen, and M. O. Krause (unpublished).

${ }^{5}$ J. M. Bizau, P. Gerard, F. J. Wuilleumier, and G. Wendin, Phys. Rev. A 36, 1220 (1987).

${ }^{6}$ Y. Sato, T. Hayaishi, Y. Itikawa, Y. Itoh, J. Murakami, T. Nagata, T. Sasaki, B. Sonntag, A. Yagishita, and M. Yoshino, J. Phys. B 18, 225 (1985).

${ }^{7}$ C. D. Caldwell, M. G. Flemming, M. O. Krause, P. van der Meulen, C. Pan, and A. F. Starace, Phys. Rev. A 41, 542 (1990).

${ }^{8}$ A. Hausmann, B. Kämmerling, H. Kossmann, and V. Schmidt, Phys. Rev. Lett. 61, 2669 (1988).

${ }^{9}$ B. Breuckmann, V. Schmidt, and W. Schmitz, J. Phys. B 9, 3037 (1976).

${ }^{10}$ D. L. Ederer, T. B. Lucatorto, and G. Mehlman, J. Opt. Soc. Am. 69, 520 (1979) and references therein.

${ }^{11}$ P. C. Deshmuhk and S. T. Manson, Phys. Rev. A 28, 209 (1983).

12Z. Altun, Phys. Rev. A 40, 4968 (1989).

${ }^{13}$ M. W. D. Mansfield and G. H. Newsom, Proc. R. Soc. London Ser. A 377, 431 (1981).

${ }^{14}$ M. O. Krause, T. A. Carlson, and P. R. Woodruff, Phys. Rev. A 24, 1374 (1981).

${ }^{15}$ M. O. Krause, T. A. Carlson, and A. Fahlman, Phys. Rev. A 30, 1316 (1984).

${ }^{16}$ R. P. Madden and K. Codling, Phys. Rev. Lett. 10, 516 (1963).

${ }^{17}$ B. W. Shore, J. Opt. Soc. Am. 57, 881 (1967).

${ }^{18}$ The Pearson-7 function has the form $f(x)$ $=a\left[1+(x-b)^{2} /\left(c^{2} d\right)\right]^{-d}$ where $a$ gives the amplitude of the peak, $b$ the position of the peak centroid, $c$ the nominal half width half maximum of the peak, and $d$ the "shape" of the peak. For $d=1$, the function is identically Lorentzian. In the limit as $d$ approaches infinity the function becomes identically Gaussian. For all practical purposes, however, when $d \geq 20$ the line shape is essentially Gaussian.

${ }^{19}$ H. Aksela, S. Aksela, H. Pulkkinen, G. M. Bancroft, and K. H. Tan, Phys. Rev. A 37, 1798 (1988).
${ }^{20}$ H. Aksela, S. Aksela, H. Pulkkinen, G. M. Bancroft, and K. H. Tan, Phys. Rev. A 33, 3876 (1986).

${ }^{21}$ H. Aksela, S. Aksela, H. Pulkkinen, and A. Yagishita, Phys. Rev. A 40, 6275 (1989)

${ }^{22}$ H. Aksela, S. Aksela, G. M. Bancroft, K. H. Tan, and H. Pulkkinen, Phys. Rev. A 33, 3867 (1986).

${ }^{23}$ The normalization of Fig. 4(a) was carried out in a slightly different manner. Because our CIS spectrum for the $3 \mathrm{~s}$ line was not recorded beyond $60 \mathrm{eV}$, we could not make a direct comparison between it and the off-resonance PES spectrum recorded at $80 \mathrm{eV}$. Thus, in order to normalize the PES spectra with respect to the $3 s$ CIS spectrum we made use of the theoretical cross section of the $3 s$ line from Ref. 11. According to that work, the off-resonance cross section at $54 \mathrm{eV}$ is approximately 1.7 times stronger than the cross section at 80 $\mathrm{eV}$.

${ }^{24}$ C. E. Moore, Atomic Energy Levels, Natl. Bur. Stand. (U.S.) Circ. No. 467 (U.S. GPO, Washington, D.C., 1949).

${ }^{25} \mathrm{We}$ are able to distinguish between the $3 d$ and $4 s$ satellites because we measure a small shift in energy in the position of the $3 d+4 s$ satellite in the spectrum recorded at the $2 p_{3 / 2} \rightarrow 3 d$ excitation [Fig. 4(e)] when compared to the spectrum recorded at the $2 p_{3 / 2} \rightarrow 4 s$ excitation [Fig. 4(b)]. This shift is enough to unambiguously assign it as the $3 d$ satellite in Figs. 4(e), 4(f), $4(\mathrm{~g})$, and $4(\mathrm{k})$, and the $4 s$ satellite in all other spectra.

${ }^{26}$ Based on the analysis of the spectra in Figs. 4(b) and 4(d), where we can unambiguously assign the most intense peak to the $4 s$ line and not the $3 d$, we presuppose that all excitations to $n s$ states decay into $n s$ states and not $n d$ states and vice versa, despite the lack of experimental resolution to actually resolve the higher $n d$ states from the $(n+1) s$ states.

${ }^{27}$ M. Meyer, E. V. Raven, M. Richter, B. Sonntag, R. D. Cowan, and J. E. Hansen, Phys. Rev. A 39, 4319 (1989).

${ }^{28}$ C. D. Caldwell, M. G. Fleming, M. O. Krause, and P. van der Meulen (unpublished).

${ }^{29}$ M. O. Krause and J. H. Oliver, J. Phys. Chem. Ref. Data 8, 329 (1979).

${ }^{30}$ G. S. Brown, M. H. Chen, B. Crasemann, and G. E. Ice, Phys. Rev. Lett. 45, 1937 (1980).

${ }^{31}$ This Auger energy was determined by taking the difference between the $2 p_{1 / 2}$ threshold energy from Ref. 10 and the $\mathrm{Mg}$ III ground-state energy given in Ref. 24.

${ }^{32}$ C. Froese-Fischer, Comput. Phys. Commun. 14, 145 (1978). 